\title{
TUNING PD AND PID CONTROLLERS VIA THE LAMBERT W FUNCTION FOR DOUBLE INTEGRATOR PLUS DEAD TIME PROCESSES
}

\author{
$U D C(681.5 .1: 681.5 .015)$
}

\author{
Radmila Gerov, Zoran Jovanović \\ University of Niš, Faculty of Electronic Engineering, Department of Control Systems, \\ Niš, Republic of Serbia
}

\begin{abstract}
The paper explores the Proportional-derivative controller for a double integrator plus dead time processes, which is a challenging control problem, that is designed based on the existing Proportional-integrative controller for integrator plus dead time processes. The PD controller is extended with an integral action and an ideal PID controller is received. The parameters of both controllers are received by using the pole placement technique, whereby the transcendent characteristics equation of the closed loop system is solved by using the Lambert $W$ function. The paper also examines the influence of the desired poles of the system with a closed feedback as well as the influence of the disturbance and the change of the DIPTD processes parameters onto the received control system performances. The results received by simulation, and the quantitative indicators, show that the proposed control system has better performances in comparison to the control systems obtained by other methods in literature.
\end{abstract}

Key words: PID controller, PD controller, double integrator plus dead time processes, pole placement, time delay, Lambert $W$ function

\section{INTRODUCTION}

Proportional-integrative-derivative (PID) controllers and Proportional-integrative (PI) controllers are most often used in the industry. This kind of a controller encompasses around $90 \%$ of all control loops and it could be told that PID controllers represent the bread and butter of automatic control [1]. Since 1942, when the Ziegler-Nichols procedure of designing a PID controller has been first introduced [2] numerous different methods for designing them have been developed. A significant number of this kind of designing procedures has been published by O'Dwyer [3].

Received August 15, 2018

Corresponding author: Zoran D. Jovanović

Faculty of Electronic Engineering, Aleksandra Medvedeva 14, 18000 Niš, Republic of Serbia

E-mail: zoran.jovanovic@elfak.ni.ac.rs 
It is commonly known that the mathematical model of the system represents the idealization of the real process or the control system. The reality has shown that, for the purposes of designing a controller, it is enough that the considered process is identified by using the model of the lower-order system [4]. There are two groups of models of the lower-order system which could be used for describing the real process: self-regulating process models and non-self-regulating process models. The former include, inter alia: the first-order plus time delay (FOPTD) process model and the second-order plus time delay (SOPTD) process model. The latter encompass the open loop unstable process models, among which there is an integrator plus dead time (IPTD) process model and the double integrator plus dead time (DIPTD) process model.

Apart from mathematical system models, the presence of time delays, or dead times, exists in a wide range of industrial systems, such as neural networks, biological systems, and chemical processes. The time delay in real processes and systems occurs as a consequence of the transmission of mass, energy or information, e.g. the central heating system of a building, the remote control of vehicles. In addition, the complex industrial systems often consist of multiple serially connected simple dynamic systems, which, as a result, produce the time delay due to the accumulation of time lags or the processing time [5]. Numerous processes in the industry, apart from the time delay, have the integrative influence too, which is why their dynamics could be described by using the IPTD process model. The most typical example is a storage tank with an outlet pump, the high-pressure steam flowing to a steam turbine generator in a power plant, the heating of well-insulated batch systems, and the batch preparation of solutions by addition of chemicals to solvent [6]. DIPTD process model can be used to describe, for example, the single axis spacecraft rotation, rotary crane motion, dynamic positioning systems of ships and other vessels [7].

The time delay can be the cause of poor performances and the control systems instability, which is why the analysis of stability is extremely important in [8], among the rest. For eliminating long dead time, compensators such as the Smith predictor [9] are used, whereby the control systems with the Smith predictor are sensitive to the system parameter changes, especially to the time delay. More optimal system performances with closed loop feedback, i.e. more robust control systems are received in literature by using the common modifications of the Smith predictor, inter alia, by using the modification given in [10]. For controlling the processes and systems with a low time delay, the PI controllers and PID controllers are used, for their use yields good performances of the closed loop system and simultaneously the control system is more robust to the changes of the process parameters in comparison to the control system with the Smith predictor.

The presence of a time delay significantly complicates the procedure of designing the controller by using more traditional methods in the time and frequency domain. To avoid solving the transcendent characteristic closed loop system equation, when designing the controller, the time delay is most often approximated by using the Padé approximation or Taylor's approximation.

In this paper, a PD controller for the DIPTD systems is designed according to the existing Proportional-integral (PI) controller for IPTD processes [11]-[12]. It is well known that the application of the PD controller with DIPTD processes, in case of disturbance, yields the off-set of the output signal. The off-set of the output signal could be eliminated by using the controller with the integrative effect, i.e. by using the PID controller. 
With the purpose of designing a PD controller for the DIPTD systems, as well as designing a PID controller, in this paper, the pole placement technique has been used. The proposed method enables the solution of the transcendent system equation, for the desired poles of the closed loop system, without the approximation of the time delay, by employing the features of the Lambert $\mathrm{W}$ function in the scalar and matrix form [13][20]. The results have been obtained by using the LambertW_DDE_Toolbox [21].

The influence of the desired poles onto the robustness of the received control systems has been examined by comparing the obtained data for: $M_{s}$-sensitivity index, $G M$-gain margin, $P M$-phase margin. The influence of the DIPTD process parameters change onto the closed loop system response has been considered, too. The performances of the received control systems with the PD and PID controllers for different desired poles, in the transition period, have been compared by measuring the settling time of the obtained closed loop system output signal and the overshoot of the output signal expressed in percentages for the set-point response and in the deviation of the output signal from the reference signal for the disturbance response. In Matlab, the received results are compared with the results obtained by using other methods given in literature.

This paper is organized in the following way. In Section 2, there is a short description of the Lambert W function. The development of the PD controller and simulation results are presented in Section 3, while in the next section, the PID controller is developed. The comparison of the received results is given in Section 5. The concluding remarks are given in Section 6 .

\section{LAMBERT W FUNCTION}

The multivalued transcendental Lambert $\mathrm{W}$ function [13], labelled as $W(z)$, where $z$ belongs to the set of complex numbers $C$, is the solution of the equation

$$
W(z) e^{W(z)}=z
$$

This function has an infinite number of solutions, i.e. $W_{k}(z)$ branches, where $k$ denotes the ordinal number of the branch and the $k \epsilon(-\infty, \infty)$. In case that the argument $z$ belongs to the set of real numbers $R$, there are only two branches $W_{k}(z)$ which can have a real value, and that is: principal branch $W_{0}(z)$ for $k=0$ and the branch $W_{-1}(z)$ for $k=-1$. This feature of the Lambert $\mathrm{W}$ function has a key role in solving the problems related to the controller design.

The value graph of the Lambert W function, for the real value of the argument $z$, has been in Figure 1, wherefrom it can be observed that:

a) if $z=-e^{-1}=-0.3679$ both branches of the Lambert $\mathrm{W}$ function have identical values, i.e. $W_{0}(z)=-1$ and $W_{-1}(z)=-1$,

b) for $-e^{-1}<z<0$, there are two different solutions of the Lambert $\mathrm{W}$ function: the principal branch $-1<W_{0}(z)<0$, while the solution of the branch for $k=-1, W_{-1}(z)$ is within the range $(-\infty,-1)$, (for example: if $z=-0.2$, then $W_{0}(z)=-0.2592$ and $W_{-1}(z)=-$ 2.5426),

c) if $z=0$, there is merely one solution $W_{0}(z)=0$, 
d) for $z>0$, the value which the principal branch assumes is $W_{0}(z)>0$, while $W_{-1}(z)$ does not have a solution which belongs to the set of real numbers (for example: if $z=1.5$, then $W_{0}(z)=0.7259$ and $\left.W_{-1}(z)=-1.1217-4.4663 j\right)$.

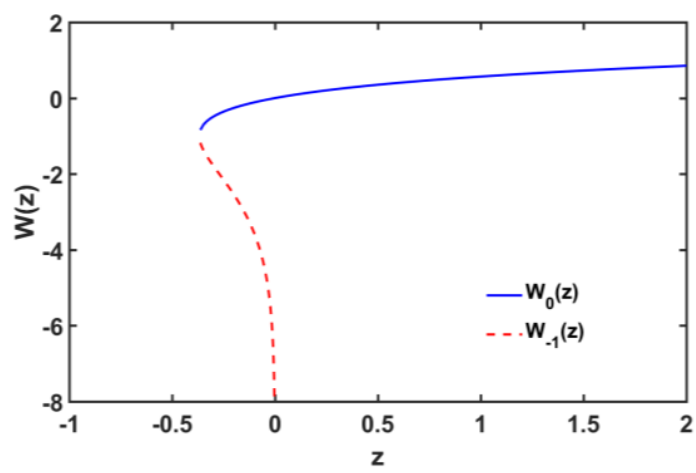

Fig. 1 Two main branches of the Lambert W function for $z \epsilon R$

The principal branch $W_{0}(z)$ is analytic at the point of zero, which ensues from the Lagrange's inversion theorem which, in turn, provides the series expansion with the radius of convergence $e^{-1}$

$$
W_{0}(z)=\sum_{n=1}^{\infty} \frac{(-n)^{n-1}}{n !} z^{n} .
$$

A more detailed explanation related to the range of branches, the manner of solution of (1), etc. could be found, except for [13] in other papers published by the same author or other authors.

\section{TUNING PD CONTROLLER FOR DIPTD PROCESSES}

Fig. 2 shows a conventional one-degree-of-freedom (1-DOF) PD control system, where: $C(s)$ - the transfer function of the PD controller, $G(s)$ - the transfer function of the DIPTD process, $r$ - the set-point, $d$ - the disturbance signal, $y$ - the controlled output, $u$ - the control signal, $e$ - the control error and $e=r-y$.

Let the transfer function of controlled DIPTD processes, where: $K$-the controlled DIPTD plant gain and $\theta$-the dead time, be

$$
G(s)=\frac{K}{s^{2}} e^{-\theta s} .
$$

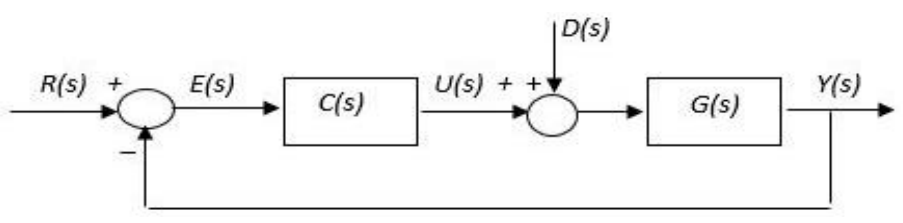

Fig. 2 Conventional 1-DOF controller control system 
The transfer function of the ideal PD controller, where: $K_{p}-$ proportional gain, and $K_{d}-$ differential gain, is

$$
C(s)=K_{p}+K_{d} s
$$

The transfer function of the real PD controller [22] is

$$
C(s)=K_{p}+K_{d} D_{s}(s),
$$

where $D_{s}(s)$ - the approximate derivative term, has the value

$$
D_{s}(s)=\frac{s}{1+T_{f} s}=\frac{s}{1+\frac{K_{d} s}{N}}, T_{f}=\frac{K_{d}}{N}, N>>1 .
$$

The controlled output from Fig. 2 is

$$
Y(s)=H_{s}(s) R(s)+H_{d}(s) D(s),
$$

where: $H_{s}(s)$ - the set-point transfer function and $H_{d}(s)$ - the load disturbance transfer function are in the form

$$
\begin{aligned}
& H_{s}(s)=\frac{C(s) G(s)}{1+C(s) G(s)}, \\
& H_{d}(s)=\frac{G(s)}{1+C(s) G(s)} .
\end{aligned}
$$

By replacing (3) and (4) into (8) and (9) the set-point transfer function and the load disturbance transfer function are received

$$
\begin{aligned}
& H_{s}(s)=\frac{K\left(K_{d} s+K_{p}\right) e^{-\theta s}}{s^{2}+K\left(K_{d} s+K_{p}\right) e^{-\theta s}}, \\
& H_{d}(s)=\frac{K e^{-\theta s}}{s^{2}+K\left(K_{d} s+K_{p}\right) e^{-\theta s}} .
\end{aligned}
$$

From (10) the transcendent characteristic equation of the control system with an infinite number of solutions is obtained

$$
\Delta(s)=s^{2}+K\left(K_{d} s+K_{p}\right) e^{-\theta s}=0 .
$$

The characteristic equation (12) can be written down in its matrix form

$$
S_{k}-A-A_{d} e^{-S_{k} \theta}=0,
$$

where $A$ and $A_{d}$ are the constant matrices assuming the dimensions $2 \times 2, S_{k}$ is the complex solution matrix (13) of the same dimensions, $k$ is the ordinal number of the branch of the Lambert W function, [11]-[12], [15]-[16] and 


$$
A=\left(\begin{array}{ll}
0 & 1 \\
0 & 0
\end{array}\right), A_{d}=\left(\begin{array}{cc}
0 & 0 \\
-K K_{p} & -K K_{d}
\end{array}\right) .
$$

If the desired poles of the system with the closed loop feedback are $\lambda_{1}$ and $\lambda_{2}$, then the solution matrix $S_{k}$ could be given in the form

$$
S_{k}=\left(\begin{array}{cc}
0 & 1 \\
-\lambda_{1} \lambda_{2} & \lambda_{1}+\lambda_{2}
\end{array}\right) .
$$

By using the limitations given by Gomez and Michiels [14], the characteristic system equation (13), can be written down in the following form

$$
\theta\left(S_{k}-A\right) e^{\theta\left(S_{k}-A\right)}=A_{d} \theta Q_{k},
$$

where $Q_{k}$ is the unknown matrix of the dimensions $2 \times 2$, which needs to satisfy the equation

$$
W_{k}\left(A_{d} \theta Q_{k}\right) e^{W_{k}\left(A_{d} \theta Q_{k}\right)+A \theta}=A_{d} \theta .
$$

By applying the matrix Lambert $\mathrm{W}$ function onto (16), the solution of the characteristic system equation of the control system (13) is obtained, in the form

$$
S_{k}=\frac{1}{\theta} W_{k}\left(A_{d} \theta Q_{k}\right)+A .
$$

By solving (17) and (18) simultaneously for the desired poles $\lambda_{1}$ and $\lambda_{2}$ of the closed loop system, the unknown matrix $Q_{k}$ and the parametric PD controller are received.

At 1-DOF controller, it is not possible to simultaneously realize the optimal set-point tracking and the disturbance response. For this reason, the tuning parameters are usually given separately; one half of the parameters serve the set-point tracking, while the other half is used for the purposes of disturbance rejection.

The gain parameters of the PD controller could be received, alongside the alreadymentioned way, based on the received gain of the PI controller for the IPTD processes [11]-[12]. In order to make an analogy between 1-DOF PD controller system for DIPTD processes and 1-DOF PI controller system for IPTD processes, let us assume that the control system shown in Fig. 2 stands for 1-DOF PI control system for IPTD processes, where: $G(s)$ is the transfer function of the IPTD processes with the same gain and time delay as DIPTD processes and $C(s)$ - the transfer function of the PI controller where $K_{p i}$ - proportional gain and $K_{i}$ - integrative gain

$$
\begin{aligned}
& G(s)=G_{I P D T}(s)=\frac{K}{s} e^{-\theta s}, \\
& C(s)=C_{P I}(s)=K_{p i}+\frac{K_{i}}{s} .
\end{aligned}
$$

By replacing (19) and (20) in (8) and (9) the set-point transfer function $H_{s p i}(s)$ and the load disturbance transfer function $H_{d p i}(s)$ is received, in the function of the parameters IPTD processes and the parameters of the PI controller 


$$
\begin{aligned}
& H_{s p i}(s)=\frac{K\left(K_{p i} s+K_{i}\right) e^{-\theta s}}{s^{2}+K\left(K_{p i} s+K_{i}\right) e^{-\theta s}}, \\
& H_{d p i}(s)=\frac{K s e^{-\theta s}}{s^{2}+K\left(K_{p i} s+K_{i}\right) e^{-\theta s}} .
\end{aligned}
$$

By comparing (10) and (21) it can be inferred that the two control systems have the identical set-point transfer functions, if and only if the proportional gain of the PD controller equal to the integrative gain of the PI controller and the differential gain of the PD controller equal to the proportional gain of the PI controller

$$
K_{p}=K_{i}, K_{d}=K_{p i} .
$$

In that case, the characteristic equations of both systems are identical, which means that the received parameters of the PI controller for IPTD processes from [11] and [12] could be applied under the condition (23) for obtaining the PD controller for DIPTD processes which has the equal gain and time delay to the one at IPTD process.

The generic form for finding the parameters of the PD controller functioning as desired poles [11] is

$$
K_{p}=\frac{\lambda_{1} \lambda_{2}}{K\left(\lambda_{1}-\lambda_{2}\right)}\left(\lambda_{1} e^{\theta \lambda_{1}}-\lambda_{2} e^{\theta \lambda_{2}}\right), K_{d}=\frac{1}{K\left(\lambda_{1}-\lambda_{2}\right)}\left(\lambda_{2}^{2} e^{\theta \lambda_{2}}-\lambda_{1}^{2} e^{\theta \lambda_{1}}\right) .
$$

For the desired, so called, aggressive tuning [12], with the poles of the closed loop systems selected by using

$$
\lambda_{1 / 2}=\frac{-0.5}{\theta} \pm \frac{0.5}{\theta} j
$$

The parameters of the PD controller are obtained by using the simple formula

$$
K_{p}=\frac{0.12075}{K \theta^{2}}, K_{d}=\frac{0.53228}{K \theta} .
$$

By reducing the imaginary part of the desired pole, whereby the real part retains the value given in (25), and by finding the parameters of the PD controller from (24), a more robust control system is received.

On the other hand, the steady state error for set-point tracking at the PI controller control system for IPTD processes is equal to zero and in case that the system is being influenced by the disturbance, which is not the case at the use of PD controller for DIPTD processes. The steady-state response of PD controller controlled system $Y_{s s}$ to the step change with amplitude $A_{r}$ of the set-point variable and to the step change with amplitude $A_{d}$ of the disturbance signal is

$$
Y_{s s}=\lim _{t \rightarrow \infty}(y(t))=\lim _{s \rightarrow 0}(s Y(s))=A_{r}+\frac{1}{K_{p}} A_{d} .
$$

If the disturbance affects the control system, from (27) it could be deduced that, if $A_{d}=0$ the steady-state error for set-point tracking does not exist, and if $A_{d} \neq 0$ the steady- 
state error and its value are directly proportional to the amplitude of the disturbance and indirectly proportional to the PD controller gain. For eliminating the steady-state error for set-point tracking it is necessary to add the integrative effect of the controller.

\section{Case Study 1}

DIPTD process is described in transfer function

$$
G(s)=\frac{1}{s^{2}} e^{-0.5 s} .
$$

Two different PD controller design processes were used for the considered DIPTD process. The first $\mathrm{PD}$ controller $\left(\mathrm{PD}_{1}\right.$ controller) is designed by means of a direct method for the desired poles of the closed loop system by using (17) and (18) or with the usage of (24). The second $\mathrm{PD}$ controller $\left(\mathrm{PD}_{2}\right.$ controller) is received by the usage of parameters of the existing PI controller which was designed for the IPTD process with the same gain and time delay as the considered DIPTD process by using (23). In order to show the influence of the desired closed loop system poles on the system performance, $\mathrm{PD}_{1}$ controller and $\mathrm{PD}_{2}$ controller were designed for different desired poles.

Assuming that the desired poles of the closed loop system are $\lambda_{I / 2}=-1 \pm 0.4 \mathrm{j}$. By solving (17) and (18) or out of (24) the $\mathrm{PD}_{1}$ controller with parameters $K_{p}=0.3401$ and $K_{d}=0.9358$ is received.

$$
P D_{1}(s)=0.3401+0.9358 s
$$

For IPTD processes with $K=1, \theta=0.5$, in [12], by using the proposed method of the aggressive tuning, for the desired poles $\lambda_{1 / 2}=-1 \pm \mathrm{j}$ (25), the PI controller (20) with parameters $K_{p i}=1.0646$ and $K_{i}=0.4830$ is developed. By applying (23), for the considered DIPTD process, the $\mathrm{PD}_{2}$ controller with parameters $K_{p}=0.4830$ and $K_{d}=1.0646$ is received

$$
P D_{2}(s)=0.4830+1.0646 s
$$

Quantitative indicators of the performances of the obtained control systems in the transition period $T_{S^{-}}$settling time, $T_{r^{-}}$rise time and $O S$ - maximum percentage overshoot and the indicators of the robustness $G M$ - the gain margin, $P M$ - the phase margin and $M_{S^{-}}$ the sensitivity index, are displayed in Table 1.

Table 1 Values $T_{s,} T_{r}, O S, G M, P M$ and $M_{s}$ for different PD controllers

\begin{tabular}{ccccccc}
\hline & $T_{s}(s)$ & $T_{r}(s)$ & $O S(\%)$ & $G M(d B)$ & $P M($ degrees $)$ & $M_{s}$ \\
\hline $\mathrm{PD}_{1}$ & 6.87 & 0.792 & 36.6 & 9.7279 & 41.4187 & 1.7293 \\
$\mathrm{PD}_{2}$ & 5.00 & 0.662 & 47.0 & 8.3562 & 35.5816 & 1.9667 \\
\hline
\end{tabular}

The unit step response of the DIPTD system is shown in Fig. 3, where the indented blue line denotes the response received with the $\mathrm{PD}_{1}$ controller, and the solid black line denotes the response with the $\mathrm{PD}_{2}$ controller. 


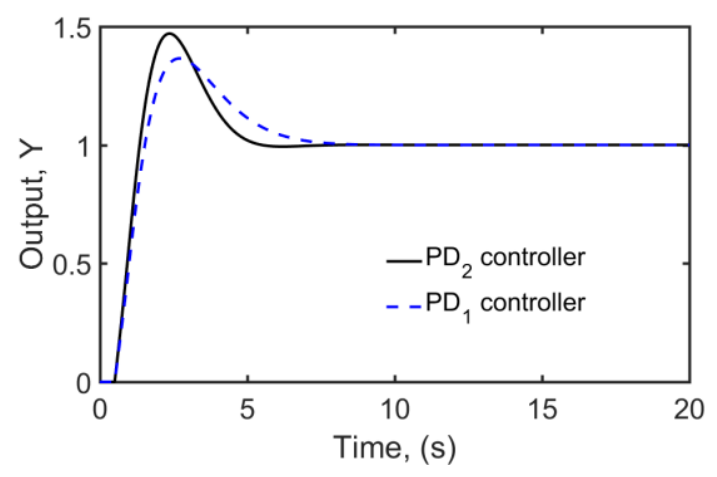

Fig. 3 Set-point response

The response of the control system under the influence of step disturbance of the unit amplitude with the reference signal equal to zero is presented in Fig. 4.

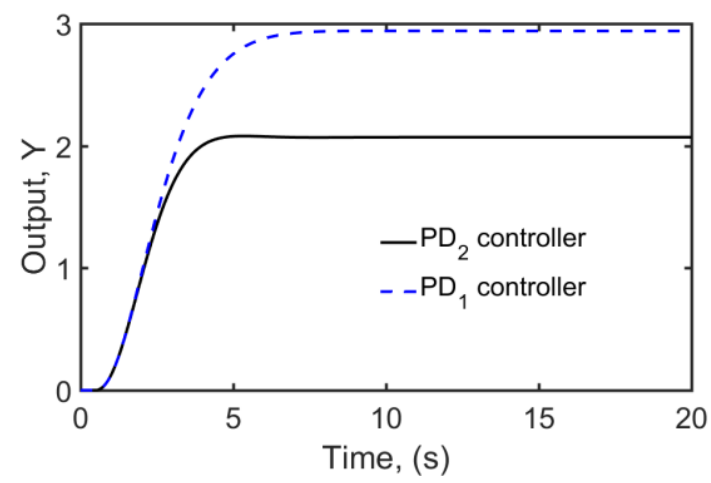

Fig. 4 Disturbance response with zero reference input

As it has already been mentioned, and which can be seen in Fig. 4, in case of the disturbance effect, there is an off-set of the output signal which can be eliminated by adding the integral effect of the controller. Based on the insight from Table 1, and on the observation of the responses given in Fig. 3 and Fig. 4, it can be inferred that the received $\mathrm{PD}_{2}$ controller by using the aggressive tuning enables faster set-point responses and faster disturbance compensation, but also the larger overshoot.

The influence of the unknown changes onto the control system, presented in the form of the change of both parameters of DIPTD processes for $30 \%$, and onto the set-point and disturbance response is shown in Fig. 5. 


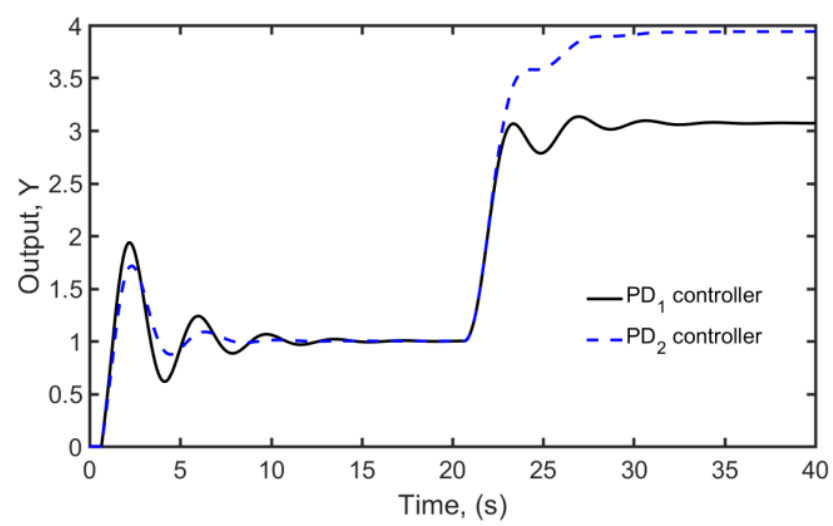

Fig. 5 Set-point and Disturbance response under perturbation in DIPTD process parameters of $30 \%$

The response from Fig. 5 and the data summed up in Table 1 clearly indicate that both control system, DIPTD process with $\mathrm{PD}_{1}$ controller and DIPTD process with $\mathrm{PD}_{2}$ controller, meet the conditions of the robustness as well as that by using the $\mathrm{PD}_{2}$ controller a better system robustness is achieved.

\section{TUNING PID CONTROLLER FOR DIPTD PROCESSES}

Let us assume that the control system shown in Fig. 2 represents 1-DOF PID control system for DIPTD processes. The transfer function of the ideal PID controller, where $K_{\text {pid }}$, $K_{i p i d}$ and $K_{d p i d}$ are gains of the proportional, integrative, and differential part of the controller, respectively, is

$$
C(s)=C_{P I D}(s)=K_{p i d}+\frac{K_{i p i d}}{s}+K_{d p i d} s .
$$

By replacing (3) and (28) into (8) and (9) the set-point transfer function $H_{\text {spid }}(s)$ and the load disturbance transfer function $H_{\text {dpid }}(s)$ are received, in the function of the parameters of DIPTD processes and the PID controller parameters

$$
\begin{aligned}
& H_{s p i d}(s)=\frac{K\left(K_{d p i d} s^{2}+K_{p p i d} s+K_{i p i d}\right) e^{-\theta s}}{s^{3}+K\left(K_{d p i d} s^{2}+K_{p p i d} s+K_{i p i d}\right) e^{-\theta s}}, \\
& H_{d p i d}(s)=\frac{K s e^{-\theta s}}{s^{3}+K\left(K_{d p i d} s^{2}+K_{p p i d} s+K_{i p i d}\right) e^{-\theta s}} .
\end{aligned}
$$

By replacing (29) and (30) in the controlled output equation (7) and by applying the final value theorem (27), the steady state error equal to zero is received. 
The characteristic equation of the received control system with the PID controller

$$
\Delta_{p i d}(s)=s^{3}+K\left(K_{d p i d} s^{2}+K_{p p i d} s+K_{i p i d}\right) e^{-\theta s}=0,
$$

Equation (31) can be expressed in the matrix shape of Equation (13), where $S_{k}$ is the complex matrix solution of the dimensions $3 \times 3$ and

$$
A=\left[\begin{array}{lll}
0 & 1 & 0 \\
0 & 0 & 1 \\
0 & 0 & 0
\end{array}\right], A_{d}=\left[\begin{array}{ccc}
0 & 0 & 0 \\
0 & 0 & 0 \\
-K K_{\text {ipid }} & -K K_{\text {ppid }} & -K K_{\text {dpid }}
\end{array}\right] .
$$

By solving (17) and (18) simultaneously for the desired poles of the closed loop system $\lambda_{1}, \lambda_{2}$ and $\lambda_{3}$, the PID controller parameters and the unknown matrix $Q_{k}$ of the dimensions $3 \times 3$, are received.

Another way of receiving the PID controller parameters (28) is using the results obtained for the PD controller. By replacing the PD controller $K_{p}$ and $K_{d}$, received in the way explained in the previous section, in the following manner

$$
K_{\text {ppid }}=K_{p}, K_{\text {dpid }}=K_{d}, K_{\text {ipid }}=\alpha K_{d}
$$

where: $\alpha$ - tuning parameter, $\alpha$ - real number slightly greater than zero, in (32) the following is received

$$
A=\left[\begin{array}{lll}
0 & 1 & 0 \\
0 & 0 & 1 \\
0 & 0 & 0
\end{array}\right], A_{d}=\left[\begin{array}{ccc}
0 & 0 & 0 \\
0 & 0 & 0 \\
-K \alpha K_{d} & -K K_{p} & -K K_{d}
\end{array}\right] .
$$

For different values $\alpha$, the received response of the closed loop system is observed and the value which satisfies the desired system performances is selected. The check of the stability of the closed loop system for different values $\alpha$ is determined by checking the obtained closed loop poles using (18), based on which the kind of given specifications of the closed loop system, such as for example the settling time, could be pointed out.

\section{Case Study 2}

The proposed method of receiving the PID controller applied to the DIPTD process given in Case Study 1 for both PD controllers.

By replacing the parameters of the $\mathrm{PD}_{1}$ controller in the formula (33) the following is received

$$
K_{\text {ppid }}=K_{p}=0.3401, K_{\text {dpid }}=K_{d}=0.9358, K_{\text {ipid }}=\alpha K_{d}=0.9358 \alpha .
$$

In Table 2, GM, $P M, M_{s}, O S$, settling time $T_{s}$ for the set-point response and the settling time $T_{s d}$ for the disturbance response are given, for the PID controllers ( PID $_{1}$ controllers) with different integrative gains received for different values of the tuning parameters $\alpha$ based on the $\mathrm{PD}_{1}$ controller. 
Table 2 Values of $G M, P M, M_{s}, O S, T_{s}$ and $T_{s d}$ for the $\mathrm{PID}_{1}$ controller received based on the $\mathrm{PD}_{1}$ controller from Case Study 1 in the function of the tuning parameter $\alpha$

\begin{tabular}{ccccccc}
\hline$\alpha$ & $T_{s}(s)$ & $T_{s d}(s)$ & $O S(\%)$ & $G M(d B)$ & PM(degrees) & $M_{s}$ \\
\hline 0.05 & 16.2 & 19.8 & 42.0 & 9.7764 & 40.7178 & 1.7179 \\
0.06 & 16.3 & 21.7 & 43.1 & 9.7864 & 40.5254 & 1.7178 \\
0.07 & 16.1 & 21.7 & 44.4 & 9.7958 & 40.3099 & 1.7159 \\
0.08 & 15.7 & 20.5 & 45.6 & 9.8051 & 40.0682 & 1.7140 \\
0.09 & 15.3 & 25.2 & 46.8 & 9.8124 & 39.7963 & 1.7120 \\
\hline
\end{tabular}

For the same values of the tuning parameter $\alpha$, Table 3 sums up the results in case that the PID controller ( $\mathrm{PID}_{2}$ controller) is designed based on the $\mathrm{PD}_{2}$ controller, i.e.

$$
K_{\text {ppid }}=K_{p}=0.4830, K_{d p i d}=K_{d}=1.0646, K_{\text {ipid }}=\alpha K_{d}=1.0646 \alpha \text {. }
$$

Table 3 Values of $G M, P M, M_{s}, O S, T_{s}$ and $T_{s d}$ for the $\mathrm{PID}_{2}$ controller received based on the $\mathrm{PD}_{2}$ controller from Case Study 1 in the function of the tuning parameter $\alpha$

\begin{tabular}{ccccccc}
\hline$\alpha$ & $T_{s}(s)$ & $T_{s d}(s)$ & $O S(\%)$ & $G M(d B)$ & PM(degrees) & $M_{s}$ \\
\hline 0.05 & 9.97 & 30.4 & 51.1 & 8.4056 & 35.1241 & 1.9564 \\
0.06 & 12.0 & 23.9 & 52.0 & 8.4149 & 35.0067 & 1.9544 \\
0.07 & 12.8 & 19.0 & 52.8 & 8.4242 & 34.8788 & 1.9523 \\
0.08 & 13.0 & 15.1 & 53.7 & 8.4332 & 34.7395 & 1.9503 \\
0.09 & 13.0 & 12.5 & 54.6 & 8.4373 & 34.5876 & 1.9485 \\
\hline
\end{tabular}

The response of the DIPTD process with parameters gain $K=1$ and dead time $\theta=0.5 \mathrm{~s}$, with different PID controllers under the influence of the disturbance in the form of the unit step function which occurs in the moment $\mathrm{t}=25 \mathrm{~s}$ and the reference unit step function signal, is shown in Fig. 6.

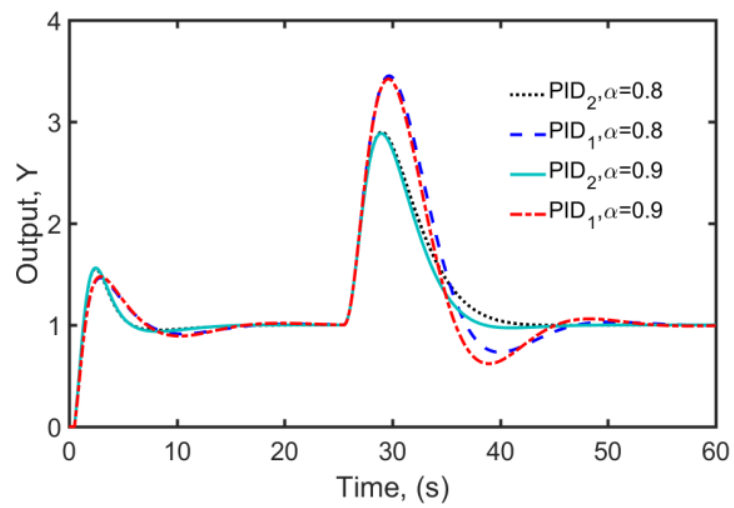

Fig. 6 Set-point and Disturbance response under perfect parameters of DIPTD process 
The response under the influence of the same disturbance and perturbation of $30 \%$ in the gain and in the time delay of the DIPTD process, is shown in Fig. 7.

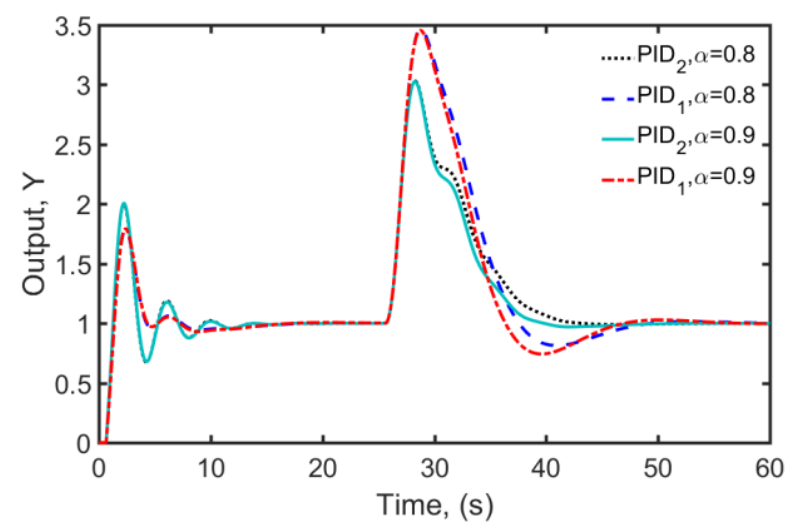

Fig. 7 Set-point and Disturbance response under perturbation of 30\% in DIPTD process parameters

According to the data from Table 2 and Table 3, it can be inferred that PID $_{1}$ controllers received based on the parameters of the $\mathrm{PD}_{1}$ controller enable better robustness of the control system compared to the $\mathrm{PID}_{2}$ controllers based on the $\mathrm{PD}_{2}$ controllers. On the other hand, by using the $\mathrm{PID}_{2}$ controllers the shorter settling time of the closed loop feedback system is obtained, as well as the faster disturbance compensation. The same conclusions could be made based on the observations from Fig. 6 and Fig. 7.

\section{SIMULATION RESULTS}

Case Study 3

The second order plus time delay (SOPTD) process model is examined, of the transfer function

$$
G(s)=\frac{40}{(20 s+1)^{2}} e^{-s}
$$

For the examined SOPTD process model Ruscio and Dalen [7] designed the ideal PID controller which is used for receiving $M_{s}=1.59$, with parameters $K_{c}=1.08, T_{i}=7.65$ and $T_{d}=3.67$, of the transfer function

$$
G_{c}(s)=K_{c}\left(1+\frac{1}{T_{i} s}+T_{d} s\right) .
$$

The examined SOPTD process model has the time constant much greater than the time delay, which is why the same could be approximated by using the DIPTD process model (3) with parameters: gain $K=0.1$ and time delay $\theta=1$.

First, by using the proposed method the PD controller is designed based on the approximated DIPTS process model. The desired poles are selected as in (25). By using the simple formula for calculating the parameters of the PD controller (26), $K_{p}=1.2075$ and 
$K_{d}=5.3228$ are obtained. After replacing the received gains in (33), for the different values of the tuning parameters $\alpha \in(0.01-0.03)$, with the step change of the 0.001 parameter, the PID controller parameters and the closed loop system response are received.

The compromise between the quicker set-point response of the closed loop system and the good robustness in terms of the faster compensation of the disturbance influence onto the system, as well as the influence of the unknown changes, is obtained for $\alpha=0.019$. By replacing the selected value of the tuning parameter $\alpha$ in (33) the integrative gain of the PID controller $K_{i}=0.1011$ is received.

The spectrum of the poles of the closed loop system, with the proposed PID controller and the SOPTD process model, is shown in Fig. 8, wherefrom it can be observed that the received control system is stable.

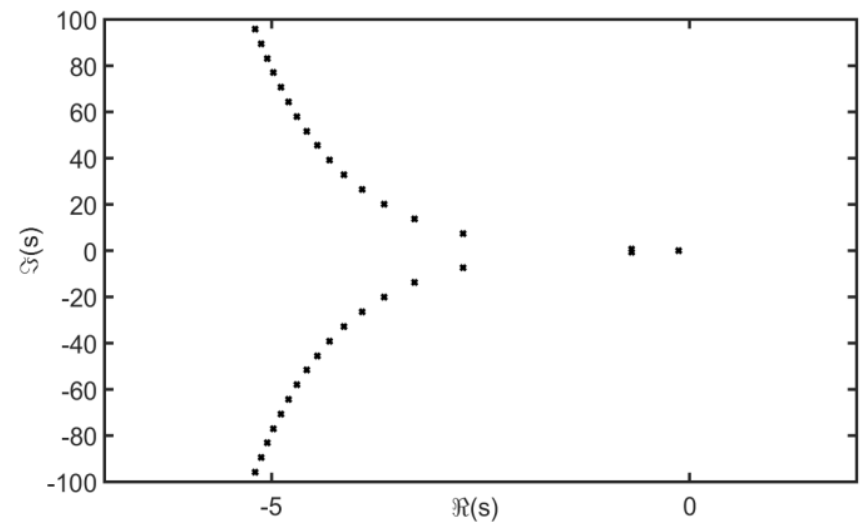

Fig. 8 Closed loop spectrum distribution

The present PID controller and the PID controller received by using Dalen and Ruscio method from [7] have been employed for controlling the examined SOPTD process model.

The set-point response for the unit step reference input without disturbance is shown in Fig. 9, where the response received by using the present-PID controller is labelled with $Y_{\text {Present }}$ while the response received by using the PID controller method from [7] is marked with $\mathrm{Y}_{\mathrm{RD}}$.

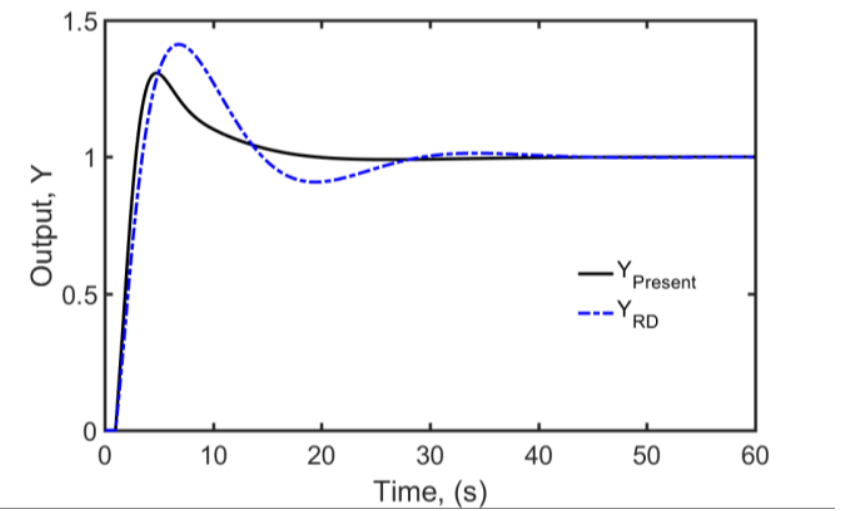

Fig. 9 Set-point response under perfect parameters of SOPTD process 
The response of the SOPTD process during the disturbance influence in the form of the unit step function, wherein the reference signal is equal to zero, with the present-PID controller and the Ruscio and Dalen-PID controller given in [7], is illustrated in Fig. 10.

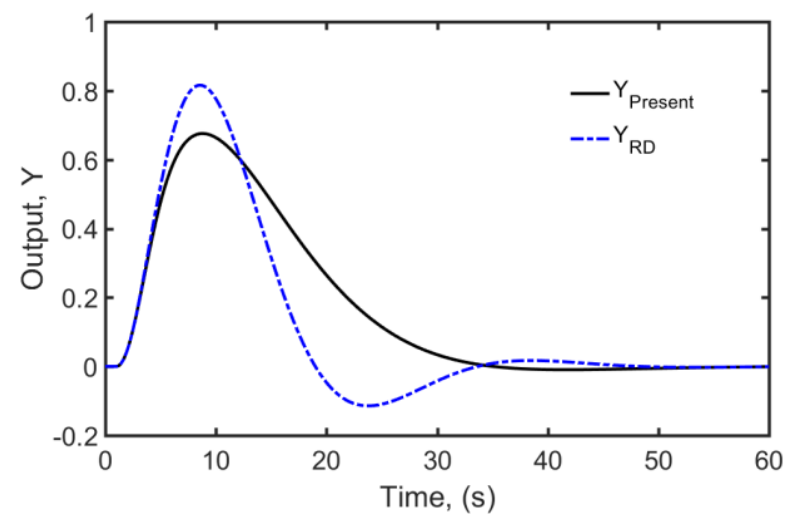

Fig. 10 Disturbance response under perfect parameters of SOPTD process

The same parameter values as in the previous section, $G M, P M, M_{s}, O S$, settling time $T_{s}$ for the set-point response and the settling time $T_{s d}$ for the disturbance response, have been used as quantitative indicators of the received results, while the results have been summed up in Table 4.

Table 4 Values of $G M, P M, M_{s}, O S, T_{s}$ and $T_{s d}$ for the PID control system for SOPTD process model in Case Study 3

\begin{tabular}{lcccccc}
\hline & $T_{s}(s)$ & $T_{s d}(s)$ & $O S(\%)$ & $G M(d B)$ & $P M($ degrees $)$ & $M_{s}$ \\
\hline Ruscio and Dalen-PID & 26.9 & 39.8 & 41.2 & 11.274 & 40.1626 & 1.5888 \\
Present-PID & 16.0 & 32.4 & 30.7 & 8.8961 & 45.4147 & 1.7677 \\
\hline
\end{tabular}

Both PID controllers satisfy the criteria of robustness. The received results indicate that the present PID controller compensates the disturbance better and that it gives better characteristics in the transition period, not only in terms of the settling time, but also in the size of the received overshoot.

The influence of the unknown changes onto the control system has been explored through the influence of the change of $+50 \%$ of all the parameters of the considered SOPTD process model (perfect parameters: process gain $K=40$, process time constant $T=20$ and process time delay $\theta=1$ ) onto the closed loop system response.

Closed loop unit step response under perturbation of $+50 \%$ in SOPTD process parameters is shown in Fig. 11, while the mentioned influence of the change onto the disturbance response given in Fig. 12.

On Fig. 11 and 12, it can be noticed that these changes at both control systems with PID controllers lead to the longer settling period and the greater overshoot, but it can also be observed that the system with the proposed PID controller indicates a lower oscillation. From this, it can be inferred that using the proposed PID controller, even in case of the unknown changes which affect the system, better performances are received. 


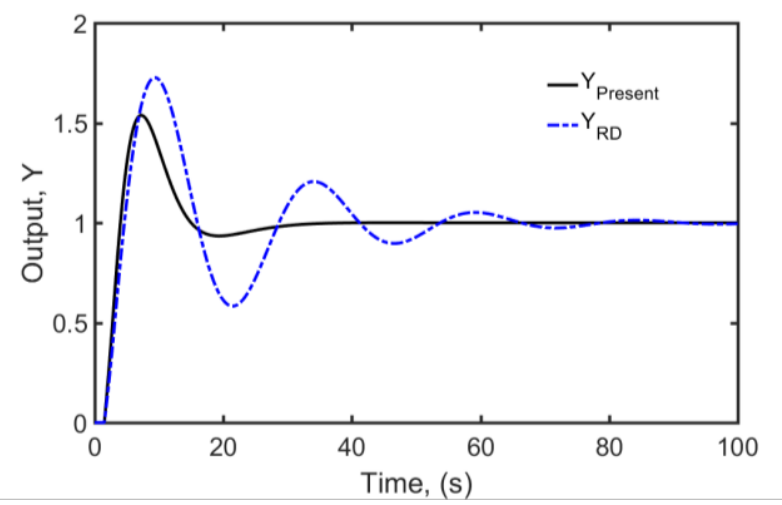

Fig. 11 Set-point response under perturbation of $+50 \%$ in SOPTD process parameters

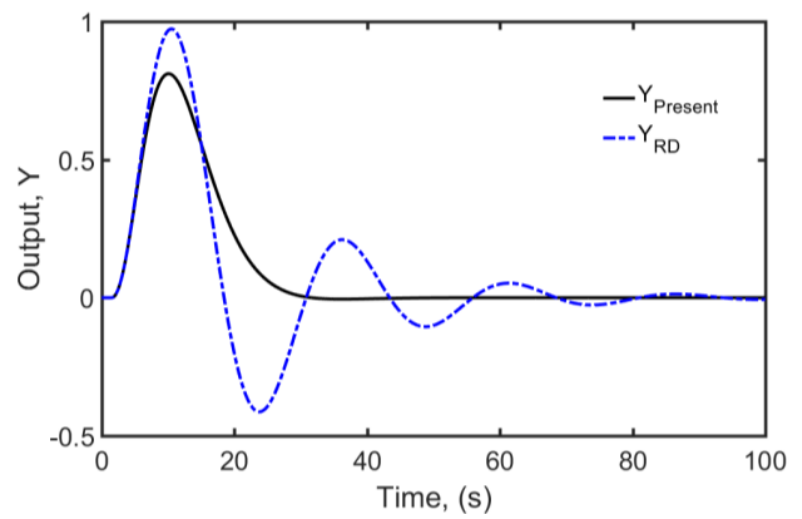

Fig. 12 Disturbance response under perturbation of $+50 \%$ in SOPTD process parameters

\section{CONCLUSION}

The paper represents two ways of designing PD and PID controller for a double integrator with dead time processes or systems. The first way is the well-known pole placement technique of designing the controller whereby the Lambert $\mathrm{W}$ function for solving the transcendent characteristic equation was applied. The other method enables the synthesis of the PD controller by using the already-received gains of the PI controller for the integrator plus dead time processes. The gains of the PD controllers within the latter proposed way of designing a PID controller are the gains of the proportional and differential part of the controller, and the integral gain is selected based on the performances for different values of the tuning parameter. The stability is examined by checking the pole locations of the closed loop system whereby the calculation is applied to the Lambert W function.

The paper has provided a simple formula for calculating the gain of the PD controller which could be applied in the teaching practice. By using the proposed method, the PID controller which enables a much faster response, and better disturbance compensation as well as better performances under the influence of the unknown changes in comparison with other methods compared is obtained. 
Acknowledgement: This paper was realized as a part of the projects "Studying climate change and its influence on the environment: impacts, adaptation and mitigation" (III 43007), and "Research and Development of New Generation Wind Turbines of High-energy Efficiency" (TR 35005), financed by the Ministry of Education, Science and Technological Development of the Republic of Serbia.

\section{REFERENCES}

[1] K. Aström, T. Hägglund, "The future of PID control," Control Engineering Practice, vol. 9, pp. 1163-1175, 2001.

[2] J. G. Ziegler, N. B. Nichols, "Optimum settings for automatic controllers", Transactions ASME, vol. 64, pp. 759$768,1942$.

[3] A. O’Dwyer, Handbook of PI and PID controller tuning rules, Imperial College Press, 3nd edition, London, U.K. 2009.

[4] S. Skogestad, "Simple analytic rules for model reduction and PID controller tuning", Journal of Process Control, vol. 13, no. 4, pp. 291-309, 2003. [Online]. Available: http://folk.ntnu.no/skoge/publications/2003/ tuningPID/finalpaper.pdf

[5] J. E. Normey-Rico, E. F. Camacho, Control of Dead-Time Processes, Springer-Verlag, London, 2007.

[6] A. Visioli, Q. C. Zhong, Control of Integral Processes with Dead Time, Advances in Industrial Control series, Springer-Verlag, London, 2011.

[7] D. D. Ruscio, C. Dalen, "Tuning PD and PID Controllers for Double Integrating Plus Time Delay Systems," Modeling, Identification and Control, vol. 38, no. 2, pp. 95-110, 2017. [Online]. Available: http://www.micjournal.no/PDF/2017/MIC-2017-2-4.pdf

[8] S. B. Stojanović, D. Lj. Debeljković, D. S. Antić, "Finite-Time stability and stabilization of linear time-delay systems," Facta Universitatis, Series: Mechanics, Automatic Control and Robotics, vol. 11, no. 1, pp. 25-36, 2012. [Online]. Available: http://casopisi.junis.ni.ac.rs/files/journals/2/olderissues/acar201201/acar20120103.pdf

[9] O. J. Smith, "Closed control of loops with dead time, Chemical Engineering Progress," vol. 53, pp. 217 219, 1957.

[10] M. S. Matijević, M. R. Stojić, "The robust controller design for processes with dead times," Facta Universitatis, Series: Mechanics, Automatic Control and Robotics, vol. 5, no 17, pp. 131 - 144, 2006. [Online]. Available:http://casopisi.junis.ni.ac.rs/files/journals/2/olderissues/macar200601/macar200601-11nn.pdf

[11] R. Gerov, Z. Jovanović, "Synthesis of PI Controller with a Simple Set-Point Filter for Unstable First-Order Time Delay Processes and Integral plus Time Delay Plant," Elektronika ir Elektrotechnika, vol. 24, no. 2, pp. 3-11, 2018. [Online]. Available: http://dx.doi.org/10.5755/j01.eie.24.2.20629.

[12] R. Gerov, Z. Jovanović, "A Simple Method of Tuning PI Controller for Integrator Dead/Time Processes," 2018 16th IEEE International Symposium on Intelligent Systems and Informatics (SISY), pp. 00051-00056, 2018.

[13] R. Corless, G. Gonnet, D. Hare, D. Jeffrey, D. Knuth, "On the Lambert W function," Advances in Computational Mathematics, vol. 5, pp. 329-359, 1996.

[14] R. C. Gomez, W. Michiels, "Some special cases in the stability analysis of multi-dimensional time-delay systems using the matrix Lambert W function," Automatica, vol. 53, pp. 339-345, 2015.

[15] S. Yi, P. W. Nelson, A. G. Ulsoy, "Analysis and Control of Time Delayed Systems via the Lambert W Function," IFAC Proceedings Volumes, vol. 41, no. 2, pp. 13414-13419, 2008. [Online]. Available: https://doi.org/10.3182/20080706-5-KR-1001.02272.

[16] S. Yi, P. W. Nelson, A. G. Ulsoy, "Eigenvalue assignment via the Lambert W function for control of time-delay systems," Journal of Vibration and Control, vol. 16, no. 7-8, pp. 961-982, 2010.

[17] S. Yi, P. W. Nelson, A. G. Ulsoy, " Proportional-Integral Control of First-Order Time-Delay Systems via Eigenvalue Assignment," IEEE Transactions on Control Systems Technology, vol. 21, no. 5, pp. 1586-1594, 2013.

[18] S. Yi, P. W. Nelson, A. G. Ulsoy, "Robust control and time-domain specifications for systems of delay differential equations via eigenvalue assignment," Journal of Dynamic Systems, Measurement and Control, vol. 132 , no. 3, pp. 031003-1 - 031003-7, 2010 .

[19] A. G. Usloy, "Time-Delayed Vibration Control of Two Degree-Of-Freedom Mechanical System for Improved Stability Margins," IFAC-PapersOnLine, vol. 48, no. 12, pp. 001-006, 2015. [Online]. Available: https://doi.org/10.1016/j.ifacol.2015.09.343.

[20] A. G. Usloy, "Time-Delayed Control of SISO Systems for Improved Stability Margins," Journal of Dynamic Systems, Measurement and Control, vol. 137, no. 4, pp. 041014-1 -041014-12, 2015. 
[21] S. Yi, S. Duan, P. W. Nelson, A. G. Ulsoy, "The Lambert W Function Approach to Time Delay systems and the LambertWDDE toolbox," IFAC Proceedings Volumes, vol. 45, no. 14, pp. 114-119, 2012. [Online]. Available: https://doi.org/10.3182/20120622-3-US-4021.00008.

[22] E. Jahanshahi, V. D. Oliveira, C. Grimholt, S. Skogestad, "A comparison between internal model control, optimal pidf and robust controllers for unstable flow in risers," IFAC Proceedings Volumes, vol. 47, no. 3, pp. 5752-5759, 2014. [Online]. Available: https://doi.org/10.3182/20140824-6-ZA-1003.02381. 